\section{RSP}

http://www.rsp.fsp.usp.br/
Revista de Saúde Pública

\title{
Tuberculose e infecção latente em funcionários de diferentes tipos de unidades prisionais
}

Péricles Alves Nogueira', Regina Maura Cabral de Melo Abrahão', Vera Maria Neder Galesi", Rossana Verónica Mendoza López"II

I Universidade de São Paulo. Faculdade de Saúde Pública. Departamento de Epidemiologia. São Paulo, SP, Brasil

" Governo do Estado de São Paulo. Secretaria de Estado da Saúde. Centro de Vigilância Epidemiológica "Prof. Alexandre Vranjac". São Paulo, SP, Brasil

III Universidade de São Paulo. Faculdade de Medicina. Instituto do Câncer do Estado de São Paulo Octávio Frias de Oliveira. Centro de Investigação Translacional em Oncologia. São Paulo, SP, Brasil

\section{RESUMO}

OBJETIVO: Estimar a prevalência de tuberculose ativa e de infecção latente da tuberculose entre funcionários contatos e não contatos de detentos, e investigar fatores associados à infecção latente da tuberculose nesta população.

MÉTODOS: Estudo observacional do tipo transversal, realizado no período de 2012 a 2015, em funcionários de diferentes unidades prisionais do município de Franco da Rocha, SP. Consistiu na aplicação de um questionário, aplicação e leitura da prova tuberculínica, baciloscopia e cultura dos escarros e exame radiológico. A associação entre as variáveis qualitativas foi calculada pelo teste qui-quadrado de Pearson e os fatores sociodemográficos e clínico-epidemiológicos relacionados à infecção latente da tuberculose foram avaliados pela regressão logística com o cálculo das odds ratios (OR) e seus respectivos intervalos com 95\% de confiança (IC95\%).

RESULTADOS: Foram examinados 1.059 funcionários, sendo 657 (62,0\%) de penitenciárias, 249 (23,5\%) de unidades da Fundação CASA e 153 (14,5\%) de hospitais de custódia e tratamento psiquiátrico. Foi aplicada e lida a prova tuberculínica em 945 (89,2\%) profissionais. Desses, 797 (84,3\%) eram contatos de detentos e 148 (15,7\%) não eram. Entre funcionários das penitenciárias, os fatores associados com a infecção latente da tuberculose foram os seguintes: ter contato com detento (OR = 2,12; IC95\% 1,21-3,71); ser do sexo masculino (OR = 1,97; IC95\% 1,19-3,27); estar na faixa etária entre 30 e 39 anos (OR = 2,98; IC95\% 1,34-6,63), 40 a 49 anos (OR = 4,32; IC95\% $1,94-9,60)$ e 50 a 59 anos (OR = 3,98; IC95\% 1,68-9,43); ser da cor ou raça não branca $(\mathrm{OR}=1,89$; IC95\% 1,29-2,78); e ser fumante ( $\mathrm{OR}=1,64$; IC95\% 1,05-2,55). Não houve exame positivo na baciloscopia e na cultura. Dos 241 (22,8\%) profissionais que realizaram o exame radiológico, 48 (19,9\%) apresentaram alterações, dos quais 11 eram suspeitos de tuberculose.

Como citar: Nogueira PA Abrahão RMCM, Galesi VMN, López RVM. Tuberculose e infecção latente em funcionários de diferentes tipos de unidades prisionais. Rev Saude Publica. 2017;51:13.

Copyright: Este é um artigo de acesso aberto distribuído sob os termos da Licença de Atribuição Creative Commons, que permite uso irrestrito, distribuição e reprodução em qualquer meio, desde que o autor e a fonte originais sejam creditados.

CONCLUSÕES: Os funcionários das penitenciárias que têm contato direto com os detentos têm 2,12 vezes mais chance de se infectar pelo Mycobacterium tuberculosis no âmbito de trabalho e, consequentemente, de adoecer por tuberculose, devendo ser alvos de ações de prevenção e controle da doença.

DESCRITORES: Tuberculose, epidemiologia. Tuberculose Latente, diagnóstico. Prisões, recursos humanos. Fatores de Risco. Condições de Trabalho. Saúde do Trabalhador. 
a Ministério da Saúde (BR),

Secretaria de Vigilância em Saúde, Departamento de Vigilância Epidemiológica. Manual de recomendações para o controle da tuberculose no Brasil. Brasília (DF); 2011 [citado 16 ago 2016]. (Série A. Normas e Manuais Técnicos).

Disponível em: http://bvsms. saude.gov.br/bvs/publicacoes/ manual_recomendacoes_controle_. tuberculose_brasil.pdf

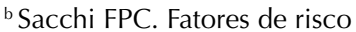
para tuberculose em população indígena, carcerária e urbana de Dourados-MS [dissertação]. Dourados: Universidade Federa da Grande Dourados; 2011.

c São Paulo. Secretaria de Estado da Saúde, Coordenação dos Institutos de Pesquisa, Centro de Vigilância Epidemiológica "Prof. Alexandre Vranjac", Divisão de Tuberculose e outras Pneumopatias. Recomendações para o controle da tuberculose nas prisões. São Paulo; 1999.

d Souza J. Sistema carcerário brasileiro: a ineficiência, as mazelas e o descaso presentes nos presídios superlotados e esquecidos pelo poder público. In: Jusbrasil [citado 18 jul 2015]. Disponível em: http://jucienesouza.jusbrasil. com.br/artigos/129905259/sistemacarcerario-brasileiro

e Ministério da Justiça (BR),

Departamento Penitenciário

Nacional. Levantamento Nacional de Informações Penitenciárias INFOPEN - junho de 2014 [citado 16 ago 2016]. Disponível em: http://www.justica.gov.br/noticias/ mj-divulgara-novo-relatorio-doinfopen-nesta-terca-feira/relatoriodepen-versao-web.pdf

' Secretaria da Administração Penitenciária do Estado de São Paulo. 165 Unidades Prisionais.

São Paulo; s.d. [citado 30 ago 2016]. Disponível em: http://www.sap.sp.gov.br

'São Paulo. Secretaria de Estado da Justiça e da Defesa da Cidadania. Fundação CASA - Centro de

Atendimento Socioeducativo ao

Adolescente. São Paulo; s.d. [citado

16 ago 2016]. Disponível em:

http://www.fundacaocasa.sp.gov.br

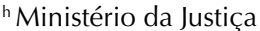

(BR), Secretaria de Justiça,

Departamento Penitenciário

Nacional. Censo Penitenciário

de 1997. Brasília (DF); 1998.

' Feitosa IMLC. Infecção latente por tuberculose em Teresina,

Piauí, Brasil: uma análise dos

casos indicados para o tratamento preventivo [dissertação]. Rio de Janeiro: Escola Nacional de Saúde Pública Sergio Arouca; 2015.

iSousa BA, Braz VHC, Paes ALV.

Prevalência de infecção por tuberculose em funcionários de um hospital universitário. [citado 18 jul 2015]. Disponível em: http://files.bvs.br/

upload/S/0101-5907/2011/

v25n1/a2587.pdf

\section{INTRODUÇÃO}

A saúde do trabalho considera a tuberculose (TB) um agravo difícil de ser controlado em locais fechados, como os ambientes prisionais, pelo fato de ser uma doença de transmissão respiratória. A aglomeração, a falta de ventilação e de iluminação natural são comuns em grande parte das unidades penais no país ${ }^{9,10,15,24,29, a, b, c}$. Segundo o Ministério da Saúde, as principais doenças identificadas nos presídios do Brasil são: TB, doenças sexualmente transmissíveis (DST), hepatite e dermatoses ${ }^{\mathrm{d}}$. Em números absolutos, o Brasil tem a quarta maior população prisional mundial, ficando atrás apenas dos Estados Unidos, da China e da Rússia, com 607.731 detentos em junho de 2014, distribuídos em 1.424 unidades prisionaise . A Secretaria de Administração Penitenciária do Estado de São Paulo possui 165 unidades prisionais distribuídas em todo o estado e a Secretaria de Estado da Justiça e da Defesa da Cidadania, 149 centros socioeducativos, denominados Fundação Centro de Atendimento Socioeducativo ao Adolescente (Fundação CASA) ${ }^{g}$.

Do total de presos brasileiros, 36\% (219.053) estão no estado de São Paulo e os coeficientes de incidência de TB nessa população atingem cerca de 800 casos por 100.000 detentos, valor bem superior aos 39,1 casos por 100.000 habitantes da população em geral do Estado ${ }^{21, e}$. Em 2014, cerca de $12 \%$ dos casos de TB no estado ocorreram na população prisional ${ }^{12}$.

Estudos realizados desde o final da década de 1990 confirmaram a elevada taxa de transmissão de TB em presídios, tanto em países desenvolvidos como em desenvolvimento, e observaram prevalência e incidência de TB muito maior na população prisional do que na população em geral ${ }^{2,4,5,10,15,25,2, \mathrm{~h}}$. Em razão disso, a OMS propôs que medidas de controle da transmissão de TB sejam adotadas nos chamados ambientes de risco, ou seja, locais que proporcionam elevada chance de infecção pelo bacilo da tuberculose ${ }^{7,824}$.

No Brasil, a incidência da doença no sistema penitenciário é 28 vezes maior do que na população geral $^{17}$. As condições de saúde nos presídios brasileiros são degradantes e transformam os detentos em um importante reservatório do bacilo da TB e, consequentemente, em fontes de infecção quando libertados ${ }^{1,3,4,10,11,22, c}$. Adicionalmente, as doenças infectocontagiosas não ficam restritas aos presídios, pois muitas são levadas para a sociedade pelo contingente de cerca de 200 mil servidores do sistema prisional que têm contato direto com a população carcerária, pelos parentes dos presos e, principalmente, pelas visitas íntimas ${ }^{2,10,17, d}$.

Portanto, os detentos e trabalhadores do sistema penal estão expostos a inúmeros riscos à saúde, podendo ser considerados integrantes de populações vulneráveis a diversas patologias 2,9,24,a,i. A TB pode ser referida como enfermidade profissional por excelência no âmbito sanitário, já havendo casos documentados da transmissão de detentos para funcionários ${ }^{2,9 . j}$. Entretanto, não existe um programa de saúde ocupacional específico, em relação à TB, visando à proteção dos profissionais do sistema prisional, que apresentam elevados índices de infecção e risco de adoecimento por essa patologia.

Diante desse contexto, o objetivo deste estudo foi estimar a prevalência de tuberculose ativa e de infecção latente da tuberculose entre funcionários contatos e não contatos de detentos, e investigar fatores associados à infecção latente da tuberculose nesta população.

\section{MÉTODOS}

Desde maio de 2011, o Centro de Vigilância Epidemiológica da Secretaria de Estado da Saúde do Estado de São Paulo, juntamente com o Departamento de Saúde da Secretaria de Administração Penitenciária e com o Departamento de Epidemiologia da Faculdade de Saúde Pública da Universidade de São Paulo, vem realizando, sob o patrocínio do Banco Mundial, um estudo intitulado "Inquérito tuberculínico do sistema prisional da área metropolitana de São Paulo", visando à elaboração de uma proposta de saúde ocupacional em tuberculose para os profissionais desse sistema. 
Este estudo foi realizado apenas em presídios da grande São Paulo. Preliminarmente, foi observado que os funcionários que tinham contato direto com os detentos apresentavam maior reatividade à prova tuberculínica, ou seja, estavam infectados pelo Mycobacterium tuberculosis. Não se conhecia se os funcionários de outros tipos de unidades prisionais apresentavam ou não o mesmo comportamento em relação à infecção latente da tuberculose (ILTB).

Como o município de Franco da Rocha possuía 12 unidades prisionais, sendo: três penitenciárias, um Centro de Detenção Provisória e um Centro de Progressão Penitenciária, pertencentes à Secretaria de Administração Penitenciária, cinco unidades da Fundação CASA e dois hospitais de custódia e tratamento psiquiátrico, foi eleito o local ideal para se conhecer a magnitude da TB pulmonar ativa e da ILTB em servidores do sistema prisional, todos funcionários públicos, doravante denominados "funcionários".

As penitenciárias são locais onde os detentos, já julgados, cumprem a sua pena em regime fechado. O Centro de Detenção Provisória abriga presos provisórios que aguardam o julgamento em regime fechado. O Centro de Progressão Penitenciária é uma unidade prisional construída junto a estabelecimentos de regime fechado, destinada a presos em regime semiabertof. As unidades da Fundação CASA oferecem assistência a jovens infratores de 12 a 21 anos incompletos, que estão inseridos nas medidas socioeducativas de privação de liberdade (internação) e semiliberdade em todo o estado de São Paulo, cuja liberação é compulsória aos 21 anos$^{\text {. }}$. Os hospitais de custódia, antigos manicômios judiciários, abrigam indivíduos julgados e considerados inimputáveis, ou seja, incapazes de responder pelos próprios atosf.

Realizou-se um estudo observacional do tipo transversal, em 1.059 funcionários das 12 unidades prisionais do município de Franco da Rocha, no período de 2012 a 2015, onde foi avaliada simultaneamente a presença de infecção latente da tuberculose em relação às variáveis de estudo e em relação às características da população.

Os seguintes critérios foram usados para a seleção de casos de TB pulmonar ativa: todo funcionário com diagnóstico confirmado por baciloscopia ou cultura e aquele em que o médico, com base nos dados clínico-epidemiológicos e no resultado de exames complementares, como o exame radiológico, firmasse o diagnóstico de TB. Como caso de ILTB, consideramos todo funcionário submetido à prova tuberculínica, cuja tuberculina usada foi o PPD-RT 23, aplicada por via intradérmica no terço médio da face anterior do antebraço esquerdo, na dose de $0,1 \mathrm{ml}$, que continha duas UT (unidades de tuberculina), e cuja leitura do maior diâmetro transverso da enduração perpendicularmente ao antebraço, após 72 horas da aplicação, e realizada com régua milimetrada transparente, apresentasse como resultado uma enduração $\geq 10$ milímetros $^{\mathrm{k}, 1}$.

k Ministério da Saúde (BR), Secretaria de Vigilância em Saúde, Departamento de Vigilância Epidemiológica. Manual nacional de vigilância laboratorial da tuberculose e outras micobactérias. Brasília (DF); 2008 [citado 16 ago 2016]. (Série A. Normas e Manuais Técnicos). Disponível em: http://bvsms.saude.gov.br/bvs/ publicacoes/manual_vigilancia_ laboratorial_tuberculose.pdf

'Ministério da Saúde (BR), Secretaria de Vigilância em Saúde, Departamento de Vigilância das Doenças Transmissíveis. Técnicas de aplicação e leitura da prova tuberculínica. Brasília (DF); 2014 [citado 16 ago 2016]. Disponível em: http://bvsms saude.gov.br/bvs/publicacoes/ tecnicas_aplicacao_leitura_ prova_tuberculinica.pdf
Os critérios de inclusão no estudo foram a adesão voluntária e o comparecimento em todas as etapas do estudo. Os de exclusão foram a recusa do funcionário em participar do estudo; o não comparecimento em qualquer etapa do levantamento dos dados, mesmo que tivesse assinado o termo de consentimento esclarecido; e aqueles que estavam em férias ou licenças médicas.

As variáveis analisadas foram classificadas em dois grupos: a) sociodemográficas: sexo, idade, estado civil, cor ou raça, nível de instrução e b) clínico-epidemiológicas: presença de tosse, tabagismo, outra doença pulmonar, tuberculose anterior, contato com os detentos, baciloscopia, cultura, radiografia de tórax e prova tuberculínica.

Quando a pesquisa foi aprovada pelos Comitês deÉtica daFaculdade deSaúdePública da Universidade de São Paulo e da Secretaria de Estado da Administração Penitenciária, houve a formação e treinamento da equipe de trabalho multiprofissional, para a coleta dos dados. Simultaneamente, ocorreu uma reunião com os diretores e com os funcionários das diferentes unidades prisionais, para elucidação dos procedimentos que seriam realizados e do impacto da tuberculose no sistema prisional, e para a elaboração de uma planilha de trabalho junto aos mesmos.

Em campo, cada funcionário que concordou em participar do estudo e que assinou o termo de consentimento livre e esclarecido, foi entrevistado, individual e sigilosamente, por um 
membro da equipe de trabalho. Na entrevista, utilizou-se um questionário já validado ${ }^{\mathrm{m}} \mathrm{e}$ que continha perguntas sobre dados sociodemográficos e clínico-epidemiológicos. Foram anotadas as seguintes informações: nome, filiação, sexo, idade, estado civil, cor ou raça, nível de instrução, tempo de trabalho na unidade prisional, setor em que trabalhava, função que ocupava, contato direto com os detentos, tuberculose anterior, contato prévio com pessoas tuberculosas, presença de tosse, expectoração, tabagismo, outra doença pulmonar, outra doença não pulmonar e se já havia feito a prova tuberculínica.

Em seguida, enfermeiras aferidas pela Secretaria de Estado da Saúde aplicaram a prova tuberculínica (PPD-RT23 - 2 UT/0,1 ml), segundo normas do Ministério da Saúdel, em todos os participantes. Foi considerado como infectado pelo $M$. Tuberculosis ou reator à prova tuberculínica o funcionário que apresentou enduração $\geq 10 \mathrm{~mm}$, segundo recomendações do Ministério da Saúde $\mathrm{e}^{\mathrm{k}, \mathrm{l}} \mathrm{e}$ do $\mathrm{CDC}^{\mathrm{n}}$. Os funcionários foram divididos em dois grupos: aqueles com enduração menor do que $10 \mathrm{~mm}$, considerados negativos; e aqueles com medida maior ou igual a $10 \mathrm{~mm}$, os positivos. Foram também classificados como "contatos" e "não contatos" de detentos.

Foram classificados como contatos os funcionários que tinham contato direto com os detentos, a saber: os agentes de segurança penitenciária (ASP); os profissionais da área da saúde; os professores; os agentes religiosos; o diretor geral; e o diretor de segurança e disciplina. Foram classificados como não contatos aqueles que não tinham contato direto com os detentos, como: os oficiais administrativos; os agentes de escolta e vigilância penitenciária (AEVP); e os funcionários da diretoria.

Realizou-se uma busca ativa de TB nessa população, sendo coletada uma amostra de escarro de cada participante, para os exames de baciloscopia e de cultura, de acordo com as recomendações do Ministério da Saúde ${ }^{k}$. As culturas positivas seriam identificadas e testadas em relação à sensibilidade às drogas antituberculose.

Os funcionários sintomáticos respiratórios detectados pelo questionário individual e os reatores à prova tuberculínica foram encaminhados para o exame radiológico de tórax e para um segundo exame de escarro. Os servidores que apresentaram anormalidades no exame radiológico foram encaminhados às unidades de saúde da região.

As variáveis qualitativas foram apresentadas pelas frequências e porcentagens. A associação entre essas variáveis foi calculada pelo teste qui-quadrado de Pearson ou teste exato de Fisher, segundo características das frequências ${ }^{19}$. Para avaliar associação entre a ILTB e as diferentes variáveis independentes, foi utilizada a regressão logística univariada e múltipla. Inicialmente, foram calculadas as odds ratio (OR) e seus respectivos intervalos de confiança de 95\% (IC95\%) para cada variável. Aquelas com valor de $\mathrm{p} \leq 0,20$ foram selecionadas para a regressão múltipla. O método stepwise backward permitiu analisar o modelo de regressão logística final com as variáveis independentes que foram estatisticamente significativas

m Abrahão RMCM. Diagnóstico da tuberculose na população carcerária dos Distritos

Policiais da Zona Oeste da Cidade de São Paulo [tese]. São Paulo: Faculdade de Saúde Pública; 2004. https://doi. org/10.11606/T.6.2004.tde03032011-144652

${ }^{\mathrm{n}}$ Centers for Disease Control and Prevention, Division of

Tuberculosis Elimination. Mantoux Tuberculosis Skin Test facilitator guide: appendix D: Mantoux Tuberculin Skin Test interpretation table. Atlanta: CDC; 2012 [citado 16 ago 2016]. Disponível em: http:// www.heartlandntbc.org/assets/ training/mini-fellowship/ PediatricToolBox/CDC/ed_ training/publications/mantoux/ appendix_D.htm e a variável de ajuste. $\mathrm{O}$ ajuste do modelo foi avaliado pelo teste de Hosmer-Lemeshow, sob a hipótese nula de que "o modelo é bom" ${ }^{19}$. O nível de significância adotado foi de $5 \%$. As análises foram realizadas no software estatístico SPSS v.18 for Windows ${ }^{\circledR}{ }^{19}$.

Este estudo foi aprovado pelos Comitês de Ética em Pesquisa da Faculdade de Saúde Pública da Universidade de São Paulo (Parecer 548.121/2014) e da Secretaria de Estado da Administração Penitenciária (Parecer 019/2011). A participação foi voluntária e todos os funcionários assinaram um termo de consentimento livre e esclarecido.

\section{RESULTADOS}

Na época da pesquisa, havia 2.143 funcionários nas 12 unidades prisionais do município de Franco da Rocha, assim distribuídos: 720 (33,6\%) nas três penitenciárias, 243 (11,3\%) no Centro de Detenção Provisória, 297 (13,9\%) no Centro de Progressão Penitenciária, 511 (23,8\%) nos dois hospitais de custódia e tratamento psiquiátrico e 372 (17,4\%) nas cinco unidades da Fundação CASA. Desses, 1.059 (49,4\%) estavam presentes e aceitaram participar do estudo. 
Dos 1.260 funcionários das cinco unidades da Secretaria de Administração Penitenciária (três penitenciárias, um Centro de Detenção Provisória e um Centro de Progressão Penitenciária), doravante denominadas "penitenciárias", 657 (52,1\%) participaram da pesquisa. Dos 372 funcionários das cinco unidades da Fundação CASA, denominadas "CASA", houve a adesão de 249 (66,9\%), e dos 511 funcionários dos dois hospitais de custódia e tratamento psiquiátrico, denominados "hospitais", houve a participação de 153 (29,9\%).

Em relação às características sociodemográficas dessa população, a distribuição dos funcionários segundo sexo, idade, estado civil, cor ou raça e nível de instrução apresentou associação significativa entre as diferentes unidades prisionais $(p<0,05)$. A maioria era do sexo masculino, da faixa etária de 30 a 49 anos, casados ou amasiados, brancos e com ensino médio de instrução. Nos hospitais, chama atenção que as mulheres representavam 50,3\% dos funcionários e que 36,0\% dos profissionais pertenciam à faixa etária de 50 a 59 anos (Tabela 1).

Quanto às características clínico-epidemiológicas dos funcionários (Tabela2), não houve associação significativa entre tosse, tabagismo ou outra doença pulmonar e unidades prisionais $(p>0,05)$.

A proporção de funcionários com tuberculose anterior diferiu entre as unidades $(\mathrm{p}<0,001)$. Nos hospitais, 25,5\% dos funcionários já haviam adoecido por TB anteriormente.

Dos 1.059 funcionários analisados, 886 (83,7\%) relataram no inquérito individual que tinham contato direto com os detentos e esse tipo de contato teve distribuição diferente entre as diversas unidades $(\mathrm{p}<0,001)$. Em todas elas, a maioria dos funcionários eram contatos, destacando-se $92,2 \%$ nos hospitais.

Tabela 1. Características sociodemográficas dos funcionários de diferentes tipos de unidades prisionais do município de Franco da Rocha, SP, 2015.

\begin{tabular}{|c|c|c|c|c|c|c|}
\hline & Fundação CASA & Hospitais & Penitenciárias & Total & & \\
\hline funcionários & $\begin{array}{c}n=249 \\
n(\%)\end{array}$ & $\begin{array}{c}n=153 \\
n(\%)\end{array}$ & $\begin{array}{c}n=657 \\
n(\%)\end{array}$ & $\begin{array}{c}n=1.059 \\
n(\%)\end{array}$ & $\chi^{2}$ & p \\
\hline Sexo & & & & & 56,95 & $<0,001$ \\
\hline Masculino & $171(68,7)$ & $76(49,7)$ & $517(78,7)$ & $764(72,1)$ & & \\
\hline Feminino & $78(31,3)$ & $77(50,3)$ & $140(21,3)$ & $295(27,9)$ & & \\
\hline Faixa etária & & & & & 52,31 & $<0,001$ \\
\hline 18 a 29 & $30(12,0)$ & $10(6,5)$ & $82(12,5)$ & $122(11,5)$ & & \\
\hline 30 a 39 & $97(39,0)$ & $40(26,1)$ & $239(36,4)$ & $376(35,5)$ & & \\
\hline 40 a 49 & $85(34,1)$ & $39(25,5)$ & $217(33,0)$ & $341(32,3)$ & & \\
\hline 50 a 59 & $35(14,1)$ & $55(36,0)$ & $100(15,2)$ & $190(17,9)$ & & \\
\hline 60 ou mais & $2(0,8)$ & $9(5,9)$ & $19(2,9)$ & $30(2,8)$ & & \\
\hline Estado civil & & & & & 15,89 & 0,014 \\
\hline Solteiro & $64(25,7)$ & $39(25,5)$ & $143(21,8)$ & $246(23,2)$ & & \\
\hline Casado/Amasiado & $156(62,7)$ & $92(60,1)$ & $446(67,9)$ & $694(65,5)$ & & \\
\hline Separado/Divorciado & $28(11,2)$ & $15(9,8)$ & $60(9,1)$ & $103(9,8)$ & & \\
\hline Viúvo & $1(0,4)$ & $7(4,6)$ & $8(1,2)$ & $16(1,5)$ & & \\
\hline Raça & & & & & 36,98 & $<0,001$ \\
\hline Branca & $128(51,4)$ & $102(66,7)$ & $472(71,8)$ & $702(66,3)$ & & \\
\hline Negra/Parda & $116(46,6)$ & $51(33,3)$ & $181(27,5)$ & $348(32,8)$ & & \\
\hline Outras & $5(2,0)$ & 0 & $4(0,7)$ & $9(0,9)$ & & \\
\hline Escolaridade & & & & & 3,38 & $<0,001$ \\
\hline Nível fundamental & $8(3,2)$ & $7(4,6)$ & $19(2,9)$ & $34(3,2)$ & & \\
\hline Nível médio & $140(56,2)$ & $85(55,5)$ & $401(61,0)$ & $626(59,1)$ & & \\
\hline Ensino superior & $101(40,6)$ & $61(39,9)$ & $237(36,1)$ & $399(37,7)$ & & \\
\hline
\end{tabular}

CASA: Centro de Atendimento Socioeducativo ao Adolescente

Valor de p menor ou igual a 0,05, em negrito, indicam que há diferenças estatisticamente significativas entre as variáveis comparadas. 
Tabela 2. Características clínico-epidemiológicas dos funcionários de diferentes tipos de unidades prisionais do município de Franco da Rocha, SP, 2015.

\begin{tabular}{|c|c|c|c|c|c|c|}
\hline Resultados & $\begin{array}{c}\text { Fundação CASA } \\
n=249 \\
n(\%)\end{array}$ & $\begin{array}{c}\text { Hospitais } \\
n=153 \\
n(\%)\end{array}$ & $\begin{array}{c}\text { Penitenciárias } \\
n=657 \\
n(\%)\end{array}$ & $\begin{array}{c}\text { Total } \\
n=1.059 \\
n(\%)\end{array}$ & $\chi^{2}$ & $p$ \\
\hline Tosse & & & & & 1,75 & 0,417 \\
\hline Sim & $42(16,9)$ & $24(15,7)$ & $89(13,5)$ & $155(14,6)$ & & \\
\hline Não & $207(83,1)$ & $129(84,3)$ & $568(86,5)$ & $904(85,4)$ & & \\
\hline Tabagismo & & & & & 4,51 & 0,105 \\
\hline Sim & $48(19,3)$ & $39(25,5)$ & $118(18,0)$ & $205(19,4)$ & & \\
\hline Não & $201(80,7)$ & $114(74,5)$ & $539(82,0)$ & $854(80,6)$ & & \\
\hline Outra doença pulmonar & & & & & 5,13 & 0,077 \\
\hline Sim & $20(8,0)$ & $9(5,9)$ & $28(4,3)$ & $57(5,4)$ & & \\
\hline Não & $229(92,0)$ & $144(94,1)$ & $629(95,7)$ & $1002(94,6)$ & & \\
\hline Tuberculose anterior & & & & & 107,7 & $<0,001$ \\
\hline Sim & $39(25,5)$ & $16(2,4)$ & $47(18,9)$ & $102(9,6)$ & & \\
\hline Não & $114(74,51)$ & $641(97,6)$ & $202(81,1)$ & $957(90,4)$ & & \\
\hline Contato com detento & & & & & 14,12 & $<0,001$ \\
\hline Sim & $141(92,2)$ & $551(83,9)$ & $194(77,9)$ & $886(83,7)$ & & \\
\hline Não & $12(7,8)$ & $106(16,1)$ & $55(22,1)$ & $173(16,3)$ & & \\
\hline Baciloscopia & & & & & NA & \\
\hline Negativa & $246(98,8)$ & $146(95,4)$ & $220(33,5)$ & $612(57,8)$ & & \\
\hline Positiva & - & - & - & - & & \\
\hline Cultura & & & & & $\mathrm{NA}$ & \\
\hline Negativa & $246(98,8)$ & $146(95,4)$ & $220(33,5)$ & $612(57,8)$ & & \\
\hline Positiva & - & - & - & - & & \\
\hline Radiografia de tórax & & & & & 1,51 & 0,471 \\
\hline Normal & $18(11,8)$ & $127(19,3)$ & $48(19,3)$ & $193(18,2)$ & & \\
\hline Alterada & $3(1,9)$ & $36(5,5)$ & $9(3,6)$ & $48(4,5)$ & & \\
\hline
\end{tabular}

CASA: Centro de Atendimento Socioeducativo ao Adolescente; NA: não se aplica

Valor de $p$ menor ou igual a 0,05, em negrito, indicam que há diferenças estatisticamente significativas entre as variáveis comparadas.

Não houve exame positivo na baciloscopia e na cultura, significando que nenhum funcionário apresentava TB pulmonar bacilífera. Chama atenção o fato de que apenas 57,8\% (612) dos funcionários aceitaram colher escarro para a realização da bacteriologia da TB. Muitos se recusaram alegando que não tinham expectoração ou qualquer outro sintoma que justificasse colher o material.

Não houve associação significativa entre o resultado do exame radiológico de tórax e as diferentes unidades prisionais $(\mathrm{p}>0,05)$. Dos $241(22,8 \%)$ profissionais que realizaram o exame, 48 (19,9\%) apresentaram as seguintes alterações: 11 imagens radiológicas suspeitas de TB, 36 suspeitas de outra pneumopatia e uma imagem com aumento de área cardíaca, sendo todos eles encaminhados às Unidades de Saúde da região. Os 11 funcionários com imagem radiológica suspeita de TB serão alvo de um novo estudo.

Foi aplicada e lida a prova tuberculínica em 945 (89,2\%) profissionais. Desses, 797 (84,3\%) trabalhavam diretamente com os detentos, ou seja, eram "contatos", e 148 (15,7\%) eram "não contatos". Os principais motivos para a não realização da prova tuberculínica nos outros 114 $(10,8 \%)$ funcionários foram a recusa e o não comparecimento à aplicação ou à leitura do teste.

Na avaliação das características sociodemográficas e clínico-epidemiológicas dos funcionários em relação à ILTB pela positividade da prova tuberculínica (PPD $\geq 10 \mathrm{~mm}$ ), observamos que, para os funcionários da Fundação CASA, o nível de instrução foi um fator associado com a ILTB ( $p=0,018$ ); as demais variáveis não mostraram associação (Tabela 3). Nos hospitais, a faixa etária foi associada com a ILTB $(\mathrm{p}=0,038)$, apresentando maior positividade ao PPD os funcionários da faixa etária de 30 a 59 anos. Funcionários que trabalhavam nas 
Tabela 3. Infecção latente da tuberculose em funcionário do sistema prisional, segundo características sociodemográficas, características clínico-epidemiológicas e local de trabalho. Franco da Rocha, SP, 2015.

\begin{tabular}{|c|c|c|c|c|c|c|}
\hline \multirow[b]{2}{*}{ Variável } & \multicolumn{2}{|c|}{ Fundação CASA } & \multicolumn{2}{|c|}{ Hospitais } & \multicolumn{2}{|c|}{ Penitenciárias } \\
\hline & $\begin{array}{c}\text { PPD }<10 \\
n(\%)\end{array}$ & $\begin{array}{c}P P D \geq 10 \\
n(\%)\end{array}$ & $\begin{array}{c}\text { PPD }<10 \\
n(\%)\end{array}$ & $\begin{array}{c}\text { PPD } \geq 10 \\
n(\%)\end{array}$ & $\begin{array}{c}\text { PPD }<10 \\
n(\%)\end{array}$ & $\begin{array}{c}\mathrm{PPD} \geq 10 \\
\text { n (\%) }\end{array}$ \\
\hline \multicolumn{7}{|l|}{ Contato com detento } \\
\hline Não & $17(40,5)$ & $25(59,5)$ & $6(66,7)$ & $3(33,3)$ & $78(80,4)$ & $19(19,6)$ \\
\hline Sim & $89(55,6)$ & $71(44,4)$ & $59(48,8)$ & $62(51,2)$ & $340(65,9)$ & $176(34,1)$ \\
\hline$p^{b}$ & & 0,080 & & 0,492 & & 0,005 \\
\hline \multicolumn{7}{|l|}{ Sexo } \\
\hline Masculino & $75(52,4)$ & $68(47,6)$ & $33(49,3)$ & $34(50,7)$ & $318(64,9)$ & $172(35,1)$ \\
\hline Feminino & $31(52,5)$ & $28(47,5)$ & $32(50,8)$ & $31(49,2)$ & $100(81,3)$ & $23(18,7)$ \\
\hline $\mathrm{p}^{\mathrm{b}}$ & & 0,990 & & 0,861 & & $<0,001$ \\
\hline \multicolumn{7}{|l|}{ Faixa etária } \\
\hline 18 а 29 & $12(54,5)$ & $10(45,5)$ & $6(100)$ & 0 & $67(89,3)$ & $8(10,7)$ \\
\hline 30 a 39 & $49(60,5)$ & $32(39,5)$ & $14(43,8)$ & $18(56,3)$ & $157(70,7)$ & $65(29,3)$ \\
\hline 40 a 49 & $31(41,3)$ & $44(58,7)$ & $17(47,2)$ & $19(52,8)$ & $126(60,9)$ & $81(39,1)$ \\
\hline 50 a 59 & $13(59,1)$ & $9(40,9)$ & $21(44,7)$ & $26(55,3)$ & $58(61,7)$ & $36(38,3)$ \\
\hline 60 ou mais & $1(50,0)$ & $1(50,0)$ & $7(77,8)$ & $2(22,2)$ & $10(66,7)$ & $5(33,3)$ \\
\hline $\mathrm{p}^{\mathrm{b}}$ & & 0,181 & & $\mathbf{0 , 0 3 8 ^ { c }}$ & & $<0,001$ \\
\hline \multicolumn{7}{|l|}{ Estado civil } \\
\hline Solteiro & $28(52,8)$ & $25(47,2)$ & $16(50,0)$ & $16(50,0)$ & $101(76,5)$ & $31(23,5)$ \\
\hline Casado/Amasiado & $68(54,8)$ & $56(45,2)$ & $39(49,4)$ & $40(50,6)$ & $276(66,7)$ & $138(33,3)$ \\
\hline Separado/Divorciado & $9(37,5)$ & $15(62,5)$ & $6(50,0)$ & $6(50,0)$ & $37(62,7)$ & $22(37,3)$ \\
\hline Viúvo & $1(100)$ & 0 & $4(66,7)$ & $2(33,3)$ & $4(50,0)$ & $4(50,0)$ \\
\hline$p^{b}$ & & $0,313^{c}$ & & $0,913^{c}$ & & 0,082 \\
\hline \multicolumn{7}{|l|}{ Cor ou Raça } \\
\hline Branca & $59(56,7)$ & $45(43,3)$ & $42(50,0)$ & $42(50,0)$ & $319(72,3)$ & $122(27,7)$ \\
\hline Negra/Parda & $46(49,5)$ & $47(50,5)$ & $23(50,0)$ & $23(50,0)$ & $95(56,9)$ & $72(43,1)$ \\
\hline Outras & $1(20,0)$ & $4(80,0)$ & 0 & 0 & $3(75,0)$ & $1(25,0)$ \\
\hline $\mathrm{p}^{\mathrm{b}}$ & & $0,202^{c}$ & & 1 & & $0,001^{c}$ \\
\hline \multicolumn{7}{|l|}{ Nível de Instrução } \\
\hline Ensino fundamental & 0 & $5(100)$ & $5(71,4)$ & $2(28,6)$ & $10(62,5)$ & $6(37,5)$ \\
\hline Ensino médio & $63(58,3)$ & $45(41,7)$ & $37(48,7)$ & $39(51,3)$ & $250(66,1)$ & $128(33,9)$ \\
\hline Ensino superior & $43(48,3)$ & $46(51,7)$ & $23(48,9)$ & $24(51,1)$ & $157(72,0)$ & $61(28,0)$ \\
\hline$p^{b}$ & & $0,018^{\mathrm{c}}$ & & $0,589^{c}$ & & 0,295 \\
\hline \multicolumn{7}{|l|}{ Tosse } \\
\hline Não & $84(51,2)$ & $80(48,8)$ & $56(50,5)$ & $55(49,5)$ & $365(68,5)$ & $168(31,5)$ \\
\hline Sim & $22(57,9)$ & $16(42,1)$ & $9(47,4)$ & $10(52,6)$ & $53(66,3)$ & $27(33,8)$ \\
\hline $\mathrm{p}^{\mathrm{b}}$ & & 0,458 & & 0,804 & & 0,690 \\
\hline \multicolumn{7}{|l|}{ Tabagismo } \\
\hline Não & $90(54,2)$ & $76(45,8)$ & $50(52,6)$ & $45(47,4)$ & $352(70,4)$ & $148(29,6)$ \\
\hline Sim & $16(45,7)$ & $19(54,3)$ & $15(42,9)$ & $20(57,1)$ & $66(58,4)$ & $47(41,6)$ \\
\hline $\mathrm{p}^{\mathrm{b}}$ & & 0,360 & & 0,323 & & 0,013 \\
\hline \multicolumn{7}{|l|}{ Outra doença pulmonar } \\
\hline Não & $99(52,7)$ & $89(47,3)$ & $60(49,6)$ & $61(50,4)$ & $398(68,3)$ & $185(31,7)$ \\
\hline Sim & $7(50,0)$ & $7(50,0)$ & $5(55,6)$ & $4(44,4)$ & $20(66,7)$ & $10(33,3)$ \\
\hline$p^{b}$ & & 0,848 & & $1^{\mathrm{c}}$ & & 0,854 \\
\hline
\end{tabular}

CASA: Centro de Atendimento Socioeducativo ao Adolescente; PPD: Derivado Proteico Purificado

a Resultado da prova tuberculínica, segundo o resultado do PPD, com ponto de corte de $10 \mathrm{~mm}$.

b Teste qui-quadrado de Pearson.

${ }^{c}$ Teste exato de Fisher.

Valor de p menor ou igual a 0,05, em negrito, indicam que há diferenças estatisticamente significativas entre as variáveis comparadas. 
penitenciárias apresentaram os seguintes fatores associados com a ILTB: contato com detento $(p=0,005)$, sexo masculino $(p<0,001)$, faixa etária $(p<0,001)$, cor ou raça $(p=0,001)$ e tabagismo $(\mathrm{p}=0,013)$.

$\mathrm{Na}$ análise dos fatores associados com a ILTB nos funcionários que trabalhavam nas penitenciárias (Tabela 4), calculou-se a OR por meio de regressão logística múltipla. Observou-se que os funcionários em contato com detentos possuem 2,12 vezes mais chances de apresentarem ILTB, comparados com aqueles funcionários que não têm contato $(\mathrm{p}=0,008)$. Adicionalmente, funcionários do sexo masculino apresentaram quase duas vezes mais chances de serem infectados quando comparados com funcionários do sexo feminino (OR = 1,97; IC95\% 1,19-3,27). Funcionários entre 30 e 39 anos, 40 a 49 anos e 50 a 59 anos apresentaram maior chance de infecção comparados com funcionários de até 29 anos. Ressalta-se que os da faixa etária de 40 a 49 anos tinham mais de quatro vezes

Tabela 4. Fatores sociodemográficos e clínico-epidemiológicos associados à infecção latente da tuberculose ${ }^{a}$ em funcionários de penitenciárias. Franco da Rocha, SP, 2015.

\begin{tabular}{|c|c|c|c|c|}
\hline \multirow{2}{*}{ Variável } & OR não ajustado & \multirow{2}{*}{$\mathbf{p}$} & OR ajustado ${ }^{b}$ & \multirow{2}{*}{$\mathbf{p}$} \\
\hline & (IC95\%) & & (IC95\%) & \\
\hline \multicolumn{5}{|l|}{ Contato com detento } \\
\hline Não & 1 & & 1 & \\
\hline Sim & $2,13(1,25-3,62)$ & 0,006 & $2,12(1,21-3,71)$ & 0,008 \\
\hline \multicolumn{5}{|l|}{ Sexo } \\
\hline Feminino & 1 & & 1 & \\
\hline Masculino & $2,35(1,44-3,84)$ & 0,001 & $1,97(1,19-3,27)$ & 0,009 \\
\hline \multicolumn{5}{|l|}{ Faixa etária } \\
\hline 18 a 29 & 1 & & 1 & \\
\hline 30 a 39 & $3,47(1,58-7,63)$ & 0,002 & $2,98(1,34-6,63)$ & 0,008 \\
\hline 40 a 49 & $5,38(2,46-11,80)$ & $<0,001$ & $4,32(1,94-9,60)$ & $<0,001$ \\
\hline 50 a 59 & $5,20(2,24-10,08)$ & $<0,001$ & $3,98(1,68-9,43)$ & 0,002 \\
\hline 60 ou mais & $4,19(1,14-15,37)$ & 0,031 & $2,90(0,75-11,12)$ & 0,122 \\
\hline \multicolumn{5}{|l|}{ Estado civil } \\
\hline Solteiro & 1 & & & \\
\hline Casado/Amasiado & $1,63(1,04-2,56)$ & 0,034 & & \\
\hline Separado/Divorciado & $1,94(1,00-3,76)$ & 0,051 & & \\
\hline Viúvo & $3,26(0,77-13,80)$ & 0,109 & & \\
\hline \multicolumn{5}{|l|}{ Cor ou Raça } \\
\hline Branca & 1 & & 1 & \\
\hline Não branca & $1,95(1,35-2,81)$ & $<0,001$ & $1,89(1,29-2,78)$ & 0,001 \\
\hline \multicolumn{5}{|l|}{ Nível de Instrução } \\
\hline Ensino fundamental & 1 & & & \\
\hline Ensino médio & $0,85(0,30-2,40)$ & 0,764 & & \\
\hline Ensino superior & $0,65(0,23-1,86)$ & 0,648 & & \\
\hline \multicolumn{5}{|l|}{ Tosse } \\
\hline Não & 1 & & & \\
\hline Sim & $1,11(0,67-1,82)$ & 0,690 & & \\
\hline \multicolumn{5}{|l|}{ Tabagismo } \\
\hline Não & 1 & & 1 & \\
\hline Sim & $1,69(1,11-2,58)$ & 0,014 & $1,64(1,05-2,55)$ & 0,029 \\
\hline \multicolumn{5}{|l|}{ Outra doença pulmonar } \\
\hline Não & 1 & & & \\
\hline Sim & $1,08(0,49-2,34)$ & 0,854 & & \\
\hline
\end{tabular}

Por meio da positividade na prova tuberculínica (PPD $\geq 10 \mathrm{~mm}$ )

${ }^{\mathrm{b}}$ Teste de Hosmer-Lemeshow para ajuste do modelo $\chi^{2}=3,15 ; \mathrm{p}=0,925$, sob a hipótese nula de que o modelo é bom. Valor de p menor ou igual a 0,05, em negrito, indicam que há diferenças estatisticamente significativas entre as variáveis comparadas. 
mais chances de serem infectados ( $\mathrm{OR}=4,32$; IC95\% 1,94-9,60). Outros fatores relacionados com a ILTB foram a cor ou raça não branca (OR = 1,89; IC95\% 1,29-2,78) em comparação com os funcionários de outras cores ou raças, assim como aqueles funcionários que fumavam, cuja chance de ser infectado era de 1,64 vez em comparação com aqueles que não fumavam $(\mathrm{p}=0,029)$.

\section{DISCUSSÃO}

As principais razões da baixa adesão à pesquisa, pois apenas 49,4\% dos funcionários estavam presentes e aceitaram participar do estudo, foram a dificuldade em conciliar o horário de trabalho dos funcionários com o horário das atividades dos integrantes da pesquisa, além de férias, licenças médicas e atividades externas. Outros se recusaram a participar, ou não compareceram na aplicação ou na leitura da prova tuberculínica. Houve maior adesão e interesse dos profissionais que tinham contato com os detentos, dos quais $83,7 \%$ concordaram em realizar todos os exames. O fator que explica a associação entre ser contato de detentos e a ILTB é a maior exposição ao bacilo a que estão sujeitos. Os profissionais que trabalham próximos aos detentos têm risco maior de se infectar pelo $M$. tuberculosis e adoecer por $\mathrm{TB}^{8,11,12}$.

Devido às diferentes características das unidades prisionais analisadas, houve associação entre o profissional ser contato de detentos e a ILTB somente nas penitenciárias. De fato, as demais unidades apresentam características que minimizam o risco de transmissão da doença. Nas unidades da Fundação CASA não há superlotação de adolescentes. As celas são bem ventiladas, o tempo de confinamento é menor e os adolescentes representam um grupo de menor risco para tuberculose. Já nos hospitais, não existem celas e sim enfermarias bem ventiladas. Apesar de pertencerem a grupos de maior risco para TB, detentos e doentes mentais passam o dia ao ar livre, em amplos espaços de lazer, utilizando as enfermarias só para dormir. Os que apresentam outras patologias, além da doença mental, ficam em quartos separados. Entretanto, notamos que 25,5\% dos funcionários dos hospitais tinham adoecido por TB anteriormente, possivelmente devido ao fato de que $36 \%$ deles tinham mais de 50 anos de idade e que a faixa etária de 30 a 59 anos foi associada à ILTB.

Para demonstrar que profissionais de presídios têm maior probabilidade de infecção e adoecimento por TB, Steenland et al. ${ }^{27}$ estudaram a viragem tuberculínica em profissionais de presídios do estado de Nova Iorque, EUA, após um surto de TB nos detentos, e constataram que $33 \%$ das novas viragens foram devidas à exposição ocupacional. No estudo realizado em um presídio para mulheres em Montreal, Canadá, Jochem et al. ${ }^{13}$, encontraram associação positiva entre trabalhar em presídios e ter infecção tuberculosa. Nogueira et al. ${ }^{18}$ mostraram que $62,4 \%$ dos servidores que trabalhavam diretamente com os detentos, em duas penitenciárias do estado de São Paulo, estavam infectados pelo bacilo da TB. Al-Darraji et al. ${ }^{2}$ encontraram uma prevalência de ILTB de $81 \%$ em funcionários de uma prisão de segurança máxima na Malásia.

A porcentagem de funcionários contatos de detentos que estavam infectados, nesta pesquisa, foi menor do que a encontrada nos estudos de Nogueira et al. ${ }^{18} \mathrm{e}$ de Al-Darraji et al. ${ }^{2}$. Isso pode ter ocorrido porque, além das diferentes características das unidades prisionais estudadas, as condições sanitárias dos presídios de Franco da Rocha eram muito boas.

Este estudo tem como limitações o fato de ter fornecido uma visão estática do problema, pois a coleta de dados tanto sobre a exposição e quanto sobre o desfecho ocorreram simultaneamente, dificultando o entendimento da relação temporal existente entre eles. Além disso, a prova tuberculínica possui suas próprias limitações em relação à aplicação e leitura do teste e à possibilidade de ocorrência de resultados falsamente negativos ou positivos, mesmo sendo ainda considerada o exame mais importante para o diagnóstico da ILTB no Brasil. E, finalmente, a pequena quantidade de estudos nacionais e internacionais com o mesmo objetivo e com a mesma população alvo limitou a comparação dos resultados obtidos neste trabalho. 
Existem vários fatores de risco para ILTB e TB ativa, como: sexo; idade; baixo nível socioeconômico; baixo nível de instrução; tabagismo; coinfecção HIV/TB; uso de álcool e outras drogas; desnutrição; diabetes mellitus; insuficiência renal e outras condições metabólicas; uso de corticosteroides, outros imunossupressores e quimioterápicos; entre outros. Esses fatores estão exacerbados em um ambiente de confinamento com superlotação, pouca ventilação e iluminação, e de baixo padrão de higiene como os presídios brasileiros ${ }^{5,7,9,16,17,23,29, b, i}$.

Neste estudo, os fatores associados à ILTB nos funcionários que trabalhavam nas penitenciárias foram, além de ter contato com detentos, ser do sexo masculino, estar na faixa etária de 30 a 59 anos, ser da cor ou raça não branca e ser fumante.

Sabe-se que a prevalência de TB é maior em adultos do sexo masculino na proporção de 2:1 a 3:1 em relação ao sexo feminino, provavelmente devido a hábitos e comportamentos mais relacionados ao homem e que também interagem na infecção pelo $M$. tuberculosis, como o abuso de álcool e tabaco, uso de drogas e exposição ocupacional a agressores inalantes. Além disso, os homens acessam menos os serviços de saúde, diminuindo a chance de diagnóstico precoce de $\mathrm{TB}^{28,31, b, o}$. Em 2012, o sexo masculino apresentou um coeficiente de incidência de $50,2 / 100.000$ hab. (2,1 vezes maior que o do sexo feminino) . $^{\mathrm{p}}$

Existem grandes diferenças na incidência da TB, relacionadas à idade. Em países menos desenvolvidos, a população mais acometida é de adultos jovens, refletindo uma transmissão recente. A doença é mais frequente na faixa etária entre 25 e 34 anos e a maior taxa de incidência ocorre na faixa entre 45 a 54 anos de idade ${ }^{31,0}$.

Em relação à cor ou raça, as pessoas pretas e pardas têm maior risco de adoecer ou morrer por tuberculose quando comparadas com as pessoas de cor branca. No Brasil, 14,1\% da população preta ou parda está entre os $10 \%$ mais pobres do país. A vulnerabilidade social, possivelmente, justifica o maior risco de adoecerem ou morrerem por TBq.

O tabagismo já foi identificado como um fator de risco para a TB desde 1918. Uma revisão sistemática (conduzida pela OMS e pela União Internacional Contra a Tuberculose e Doenças Pulmonares, The Union) confirmou a associação entre o uso do tabaco e TB infecção, TB doença, recidiva da TB e mortalidade pela doença, pois o tabaco altera a função mucociliar e de filtração de substâncias inaladas, promovendo a aderência de bactérias às células epiteliais das vias aéreas, aumentando a permeabilidade alveolar e reduzindo a imunidade humoral mediada pelas células ${ }^{14,16,20,28, a, i}$. No estudo de Al-Darraji et al. ${ }^{2}$, a ILTB foi associada com o tempo de trabalho do funcionário na prisão da Malásia e ao tabagismo. Os que fumavam apresentaram chance de 1,9 vezes de serem infectados; resultado semelhante ao encontrado neste estudo.

Considerando o elevado risco de TB nas prisões, a Secretaria de Estado da Saúde de São Pauloc $^{c}$ a Organização Mundial da Saúde ${ }^{9,30}$ e o Centers for Disease Control and Prevention $(\mathrm{CDC})^{6}$ recomendaram um maior controle educativo e preventivo aos profissionais que trabalham diretamente com os detentos, como profissionais de saúde, guardas, professores

- Ministério da Saúde (BR). Secretaria de Vigilância em Saúde. Especial Tuberculose. Bol Epidemiol. 2012;43:1-12.

${ }^{\mathrm{p}}$ Ministério da Saúde (BR), Secretaria de Vigilância em Saúde. O controle da tuberculose no Brasil: avanços, inovações e desafios. Bol Epidemiol. 2014;45(2):1-13. "Ministério da Saúde (BR), Secretaria de Vigilância em Saúde. Indicadores de Vigilância em Saúde, analisados segundo a variável raça/cor. Bol Epidemiol. 2015;46(10):1-13. etc. Se as ações para o enfrentamento da TB nas unidades prisionais não forem eficazes, será inviável o seu controle na comunidade ${ }^{9,17,30}$.

A Estratégia STOPTB da Organização Mundial da Saúde visa a "um mundo livre de tuberculose" e um dos alvos é eliminar a TB como problema de saúde pública (um caso por milhão de habitantes) até $2050^{9,31}$.

Este estudo sugere que os funcionários do sistema prisional, especialmente os que trabalham em penitenciárias e que têm contato com os detentos, devem receber treinamento adequado sobre prevenção e controle da TB, participando de um programa de saúde ocupacional com exames periódicos para detecção da infecção e da doença. A investigação da ILTB deve ser realizada nos exames admissionais e periódicos (anualmente) por meio da prova tuberculínica. Caso esteja infectado, deverá ter acesso ao tratamento para ILTB. Os funcionários com sinais 
ou sintomas compatíveis com TB ativa deverão procurar auxílio médico e serem submetidos a exames laboratoriais e à radiografia de tórax.

Pelo alto risco que apresentam de se infectar e adoecer pela TB, os funcionários do sistema prisional deveriam ser alvo de uma atenção especial por parte das autoridades em saúde e incluídos na agenda das políticas públicas. Adicionalmente, o controle do tabagismo poderia ser integrado ao programa de controle da TB nas prisões.

Os resultados deste estudo podem contribuir para a elaboração de uma proposta de saúde ocupacional em tuberculose para os profissionais do sistema prisional.

\section{REFERÊNCIAS}

1. Abrahão RMCM, Nogueira PA, Malucelli I. Tuberculosis in county jail prisoners in western sector of the city of São Paulo, Brazil. Int I Tuberc Lung Dis. 2006;10(2):203-8.

2. Al-Darraji HAA, Tan C, Kamarulzaman A, Altice FL. Prevalence and correlates of latent tuberculosis infection among employees of a high security prison in Malaysia. Occup Environ Med. 2015;72(6):442-7. https://doi.org/10.1136/oemed-2014-102695

3. Bick JA. Infection control in jails and prisons. Clin Infect Dis. 2007;45(8):1047-55. https://doi.org/10.1086/521910

4. Braun MM, Truman Bl, Maguire B, DiFerdinando GT Jr, Wormser G, Broaddus R, et al. Increasing incidence of tuberculosis in a prison inmate population. JAMA. 1989;261(3):393-7. https://doi.org/10.1001/jama.1989.03420030067031

5. Carbone ASS, Paião DSG, Sgarbi RVE, Lemos EF, Cazanti RF, Ota MM, et al. Active and latent tuberculosis in Brazilian correctional facilities: a cross-sectional study. BMC Infect Dis. 2015;15:24. https://doi.org/10.1186/s12879-015-0764-8

6. Centers for Disease Control and Prevention. Prevention and control of tuberculosis in correctional and detention facilities: recommendations from CDC. MMWR Recomm Rep. 2006 [citado 19 jul 2015];55(RR-9):1-44. Disponível em: https://www.cdc.gov/mmwr/preview/mmwrhtml/rr5509a1.htm

7. Centers for Disease Control and Prevention; National Center for HIV/AIDS, Viral Hepatitis, STD, and TB Prevention, Division of Tuberculosis Elimination. Latent tuberculosis infection: a guide for primary health care providers. Atlanta: CDC; 2013 [citado 22 nov 2016]. Disponível em: http://www.cdc.gov/tb/publications/ltbi/pdf/targetedltbi.pdf

8. Conde MB, Melo FAF, Marques AMC, Cardoso NC, Pinheiro VGF, Dalcin PTR, et al. III Diretrizes para Tuberculose da Sociedade Brasileira de Pneumologia e Tisiologia. I Bras Pneumol. 2009;35(10):1018-48. https://doi.org/10.1590/S1806-37132009001000011

9. Dara M, Grzemska M, Kimerling ME, Reyes H, Zagorskiy A. Guidelines for control of tuberculosis in prisons. Washington (DC): USAID; TBCTA; ICRC; 2009 [citado 18 nov 2016]. Disponível em: http://pdf.usaid.gov/pdf_docs/Pnadp462.pdf

10. Dara M, Acosta CD, Melchers NVSV, Al-Darraji HAA, Chorgoliani D, Reyes H, et al. Tuberculosis control in prisons: current situation and research gaps. Int I Infect Dis. 2015;32:111-7. https://doi.org/10.1016/j.jijid.2014.12.029

11. Diuana V, Lhuilier D, Sánchez AR, Amado G, Araújo L, Duarte AM, et al. Saúde em prisões: representações e práticas dos agentes de segurança penitenciária no Rio de Janeiro, Brasil. Cad Saude Publica. 2008;24(8):1887-96. https://doi.org/10.1590/S0102-311X2008000800017

12. Galesi VMN, Fukasava S. Situação epidemiológica da tuberculose no Estado de São Paulo. Bol Epidemiol Paulista. 2015;12(134):13-20.

13. Jochem K, Tannenbaun TN, Menzies D. Prevalence of tuberculin skin test reactions among prison workers. Can J Public Health. 1997;88(3):202-6. https://doi.org/10.17269/cjph.88.947

14. Lindsay RP, Shin SS, Garfein RS, Rusch MLA, Novotny TE. The association between active and passive smoking and latent tuberculosis infection in adults and children in the United States: results from NHANES. PLoS One. 2014;9(3):e93137. https://doi.org/10.1371/journal.pone.0093137 
15. Machado JC, Boldori JDM, Dalmolin MD, Souza WC, Bazzanella SL, Birkner WMK. A incidência de tuberculose nos presídios brasileiros: revisão sistemática. Rev Aten Saude. 2016;14(47):84-8. https://doi.org/10.13037/ras.vol14n47.3256

16. Narasimhan P, Wood J, Maclntyre CR, Mathai D. Risk factors for tuberculosis: review article. Pulm Med. 2013;2013:828939. https://doi.org/10.1155/2013/828939

17. Navarro PD, Almeida IN, Kritski AL, Ceccato MG, Maciel MMD, Carvalho WS, et al. Prevalência da infecção latente por Mycobacterium tuberculosis em pessoas privadas de liberdade. I Bras Pneumol. 2016;42(5):348-55. https://doi.org/10.1590/S1806-37562016000000001

18. Nogueira PA, Abrahão RMCM, Galesi VMN. Infecção tuberculosa latente em profissionais contatos e não contatos de detentos de duas penitenciárias do Estado de São Paulo, Brasil, 2008. Rev Bras Epidemiol. 2011;14(3):486-94. https://doi.org/10.1590/S1415-790X2011000300013

19. Pagano M, Gauvreau K. Princípios de bioestatística. São Paulo: Pioneira Thomson Learning; 2004.

20. Rabahi MF. Tuberculose e tabagismo. Pulmão RJ. 2012 [citado 21 nov 2016];21(1):46-9. Disponível em: http://www.sopterj.com.br/profissionais/_revista/2012/n_01/11.pdf

21. Secretaria de Estado da Saúde de São Paulo. Tuberculose no Estado de São Paulo: indicadores de morbimortalidade e indicadores de desempenho. Bol Epidemiol Paulista. 2006;3 Supl 4:S1-3.

22. Seidler A, Nienhaus A, Diel R. Review of epidemiological studies on the occupational risk of tuberculosis in low-incidence areas. Respiration. 2005;72(4):431-46. https://doi.org/10.1159/000086261

23. Shanmuganathan R, Subramaniam ID. Clinical manifestation and risk factors of tuberculosis infection in Malaysia: case study of a community clinic. Glob J Health Sci. 2015;7(4):110-20. https://doi.org/10.5539/gjhs.v7n4p110

24. Silva GD, Nogueira MF, Barreto AJR, Gois GAS, Trigueiro JVS. Risco ocupacional de tuberculose pulmonar em instituições prisionais: um resgate bibliográfico (2000-2008). Brasília (DF); s.d. [citado 18 jul 2015]. Disponível em: http://apps.cofen.gov.br/cbcenf/sistemainscricoes/arquivosTrabalhos/118010.E8.T4044.D4AP.pdf

25. Snider DE Jr, Hutton MD. Tuberculosis in correctional institutions. JAMA. 1989;261(3):436-7. https://doi.org/10.1001/jama.1989.03420030110041

26. Stead WW. Undetected tuberculosis in prison. JAMA. 1978;240(23):2544-7. https://doi.org/10.1001/jama.1978.03290230036021

27. Steenland K, Levine AJ, Sieber K, Schulte P, Aziz D. Incidence of tuberculosis infection among New York State prison employees. Am J Public Health. 1997;87(12):2012-14.

28. Ting WY, Huang SF, Lee MC, Lin YY, Lee YC, Feng JY, et al. Gender disparities in latent tuberculosis infection in high-risk individuals: a cross-sectional study. PLoS One. 2014;9(11):e110104. https://doi.org/10.1371/journal.pone.0110104

29. Valença MS, Possuelo LG, Cezar-Vaz MR, Silva PEA. Tuberculose em presídios brasileiros: uma revisão integrativa da literatura. Cienc Saude Coletiva. 2016;21(7):2147-60. https://doi.org/10.1590/1413-81232015217.16172015

30. Word Health Organization. Tuberculosis control in prisons: a manual for programme managers. Geneva: WHO; 2000 [citado 22 nov 2016]. Disponível em: http://apps.who.int/iris/bitstream/10665/66823/1/WHO_CDS_TB_2000.281.pdf

31. World Health Organization. Global tuberculosis report 2016. Geneva: WHO; 2016 [citado 22 nov 2016]. Disponível em: http://www.who.int/tb/publications/global_report/en/

Financiamento: Banco Mundial e Fundação de Amparo à Pesquisa do Estado de São Paulo (Fapesp - Processo 2012/18613-6).

Contribuição dos Autores: Concepção e planejamento do estudo: PAN, RMCMA, VMNG. Coleta, análise e interpretação dos dados: PAN, RMCMA, VMNG, RVML. Elaboração ou revisão do manuscrito: PAN, RMCMA, VMNG. Aprovação da versão final: PAN, RMCMA, VMNG, RVML. Responsabilidade pública pelo conteúdo do artigo: PAN.

Conflito de Interesses: Os autores declaram não haver conflito de interesses. 